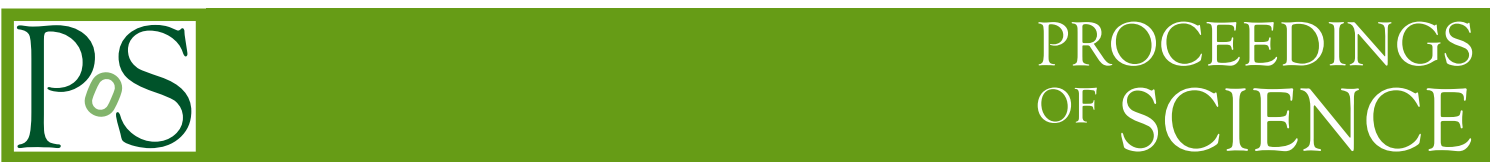

\title{
The sPHENIX experiment at RHIC
}

\section{Cameron Dean', , on behalf of the sPHENIX collaboration}

Los Alamos National Laboratory, Los Alamos, USA

E-mail: ctdean@lanl.gov

The sPHENIX experiment is the successor to the PHENIX experiment at RHIC and is optimized to study heavy flavor and jets arising from heavy ion collisions. The detector utilizes advanced technologies such as a monolithic active pixel vertex detector while also repurposing technologies originally from other high energy experiments such as BaBar, ATLAS and ALICE. The design and status of the sPHENIX detector is detailed along with the projected physics capabilities and planned measurements that the collaboration will work to achieve. sPHENIX is expected to begin data taking in 2023.

40th International Conference on High Energy physics - ICHEP2020

July 28 - August 6, 2020

Prague, Czech Republic (virtual meeting)

*Speaker 


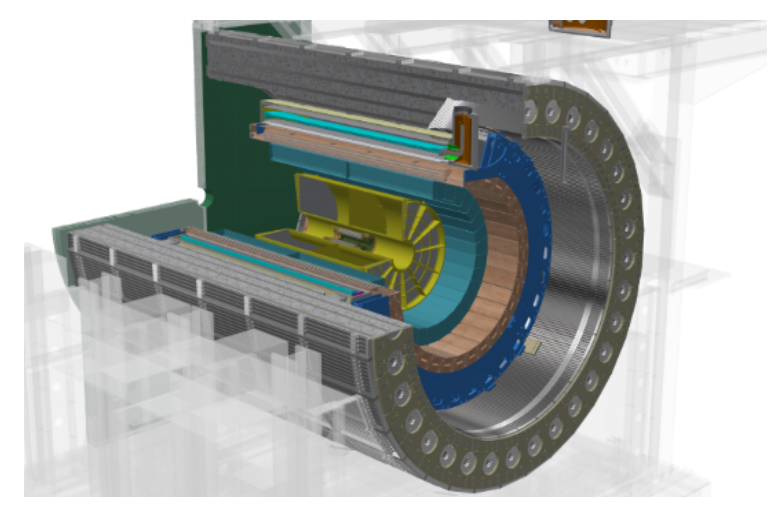

Figure 1: A mock-up of the sPHENIX detector. The MVTX and INTT detectors are in the centre of the figure, surrounded by the TPC in yellow. The EMCal surrounds the TPC in turquoise which is itself surrounded by the inner $\mathrm{HCal}$ in orange. The BaBar solenoid is shown in dark blue, surrounded by the outer HCal in grey.

\section{Introduction}

The sPHENIX experiment [1], located at the Relativistic Heavy Ion Collider at Brookhaven National Laboratory is the successor to the PHENIX experiment which will begin data taking in 2023. sPHENIX is a barrel detector, with $4 \pi$ coverage, an outer radius of $270 \mathrm{~cm}$ and a pseudorapidity range of $\eta \leq|1.1|$. The first active region is capable of taking data at $2.5 \mathrm{~cm}$ from the centre of the beam pipe. sPHENIX reconstructs vertices in the region, $\left|z_{\mathrm{vtx}}\right| \leq 10 \mathrm{~cm}$ where the $z$-axis aligns with the beam direction at a trigger rate of $15 \mathrm{kHz}$. RHIC is capable of producing AuAu collisions at $\sqrt{s_{N N}}=200 \mathrm{GeV}$ [2] and, over a three year running period, the collaboration aims to collect $1.43 \times 10^{11}$ such collisions as well as $p p$ and $p$ Au samples.

The sPHENIX detector is composed of three inner tracking layers; a three-layer monolithic active pixel vertex detector (MVTX), a two-layer intermediate silicon strip detector (INTT) and a compact time projection chamber (TPC). Further to the tracking detectors there are also two calorimeters; an electromagnetic calorimeter (EMCal) and a hadronic calorimeter (HCal) which projects to the edge of the detector volume. The hadronic calorimeter is divided into two sections called the inner and outer $\mathrm{HCal}$ which are placed on either side of the repurposed BaBar superconducting solenoid magnet [3] which operates at 1.4 T. A mock-up of the sPHENIX detector can be seen in Fig. 1.

The physics program of sPHENIX is focused on the study of charm and bottom hadrons for both heavy flavour and jet studies with the aim of simultaneously complementing the LHC program and probing regions that are unobtainable at the LHC, specifically in the low $p_{\mathrm{T}}$ region. The data sample and $p_{\mathrm{T}}$ region will allow for the study of physics topics as diverse as $D^{0}-\bar{D}^{0} \mathrm{~B}$-field correlations, jet quenching and upsilon spectroscopy which has yet to be measured at RHIC. A comparison of the physics reaches of RHIC and the LHC is given in Fig. 2.

\section{Tracking at sPHENIX}

The inner-most tracker of sPHENIX, the MVTX, consists of three layers of monolithic active pixels sensors covering a length of $27 \mathrm{~cm}$. The MVTX uses the ALICE Pixel Detector (ALPIDE) 


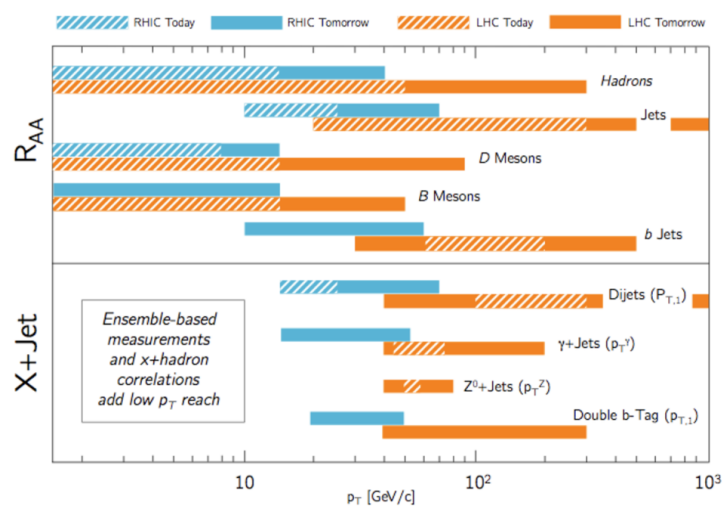

Figure 2: Comparison of the physics regions accessible by RHIC (in blue) and the LHC (in orange) in their current and future configurations, in dashed and solid line respectively.
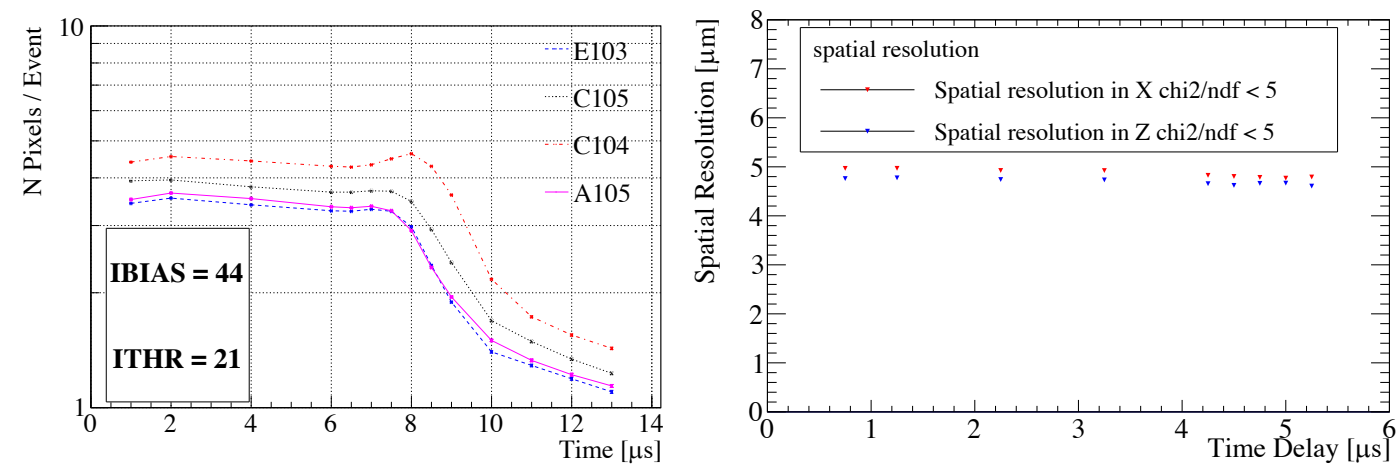

Figure 3: Left - The mean number of pixels that register a hit per event as a function of trigger delay for 4 different sensors using $120 \mathrm{GeV}$ protons. Right - The spatial resolution of the sensors as a function of trigger delay using $120 \mathrm{GeV}$ protons.

ASIC developed for use in the ALICE ITS detector [4]. The ALPIDE sensor uses $180 \mathrm{~nm}$ CMOS technology developed by TowerJazz with an overall thickness of $50 \mu \mathrm{m}$. The pixels are slightly elongated with a pitch of $29 \times 27 \mu \mathrm{m}$ and an overall matrix of $1024 \times 512$ pixels.

The mean power consumption of the ASIC is $40 \mathrm{~mW} / \mathrm{cm}^{2}$ with the detector using water cooling. To minimise the change in the flight path of particles, it is important for the MVTX to have as small a material budget as possible; the sum of the ASIC, cooling and support structure gives a perpendicular material budget of $0.3 \% X_{0}$. The MVTX has a charge integration time on the order of a few microseconds, however the exact value can be tuned by varying the pixel parameters. The time-over-threshold can be tuned to allow for the maximum hit efficiency over the period of the sPHENIX trigger latency which is approximately $5 \mu$ s and can be seen in the left of Fig. 3 The spatial resolution of the sensors has also been measured to less than $6 \mu \mathrm{m}$ for $120 \mathrm{GeV}$ protons over the full trigger latency, as seen in the right of Fig. 3.

The compact TPC used in sPHENIX will provide fast, high efficiency tracking in the region $32 \leq \mathrm{r}[\mathrm{cm}] \leq 70$ using a 50/50 mixture of Neon and $\mathrm{CF}_{4}$ and gas electron multiplier (GEM) stacks to minimise ion back flow (IBF) [5] where IBF has been measured in a TPC prototype to be less than $0.5 \%$ for GEMs biased at less than $5 \mathrm{kV}$. The TPC will be read out using the ALICE SAMPA 


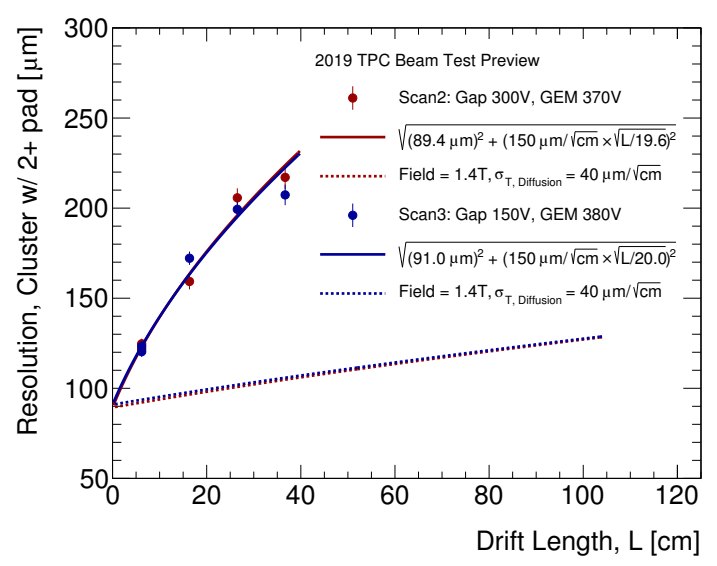

Figure 4: The spatial resolution of the TPC prototype measured using $120 \mathrm{GeV}$ protons for different GEM biases. The data points show the measured resolutions for different drift lengths, the solid lines show the fitted resolution while the dashed lines show the fit extrapolated to a magnetic field strength of $1.4 \mathrm{~T}$.

chips [6] which uses $130 \mathrm{~nm}$ technology. Each SAMPA chip contains 32 channels, each connected to a charge sensitive amplifier. The SAMPAs in sPHENIX will utilise a 80 ns peaking time in comparison to the $160 \mathrm{~ns}$ peaking time used by the ALICE TPC due to the faster drift velocity in the sPHENIX TPC.

To resolve the $\Upsilon(\mathrm{nS})$ spectrum, a mass resolution of $<100 \mathrm{MeV}$ is required. This in turn requires the TPC to have a spatial resolution of $<200 \mu \mathrm{m}$ with the maximum path length a track can have being $1 \mathrm{~m}$. A TPC prototype was produced and tested using $120 \mathrm{GeV}$ protons. The results of the test were extrapolated to measure the expected spatial resolution in the $1.4 \mathrm{~T}$ magnetic fields that sPHENIX will experience and it was found that the spatial resolution should be $<150 \mu \mathrm{m}$ for a drift length of $1 \mathrm{~m}$ as can be seen in Fig. 4.

\section{Calorimetry at sPHENIX}

The sPHENIX EMCal is a sampling calorimeter consisting of scintillating fibres embedded in a mixture of tungsten powder and epoxy [7] to give a radiation length of $20.1 \mathrm{X}_{0}$ and an interaction length of $0.83 \lambda_{\text {int }}$. The EMCal is constructed in towers, with a $2 \times 2$ set of towers constituting an EMCal block. Each tower is read out via a Hamamatsu MPPC SiPM. The blocks are tapered to allow for stereoscopic reconstruction. ${ }^{1}$ The EMCal is designed to give an energy resolution of $\sigma / \mathrm{E} \leq 16 \% / \sqrt{\mathrm{E}} \oplus 5 \%$ which has been confirmed using electrons down to an energy of approximately $2 \mathrm{GeV}$ as can be seen in the left of Fig. 5 .

Likewise, the hadronic calorimeter is a sampling calorimeter built in the shashlik style and is divided into two sections on either side of the solenoid magnet. The inner HCal uses layers of aluminium and scintillator while the outer $\mathrm{HCal}$ replaces the aluminium with steel while both layers are read out using the SAMPA chip. The interaction length is designed to be $3.8 \lambda_{\text {int }}$ for the outer $\mathrm{HCal}$ with an energy resolution of approximately $\sigma / \mathrm{E} \leq 88 \% / \sqrt{\mathrm{E}} \oplus 12 \%$. The outer HCal also

\footnotetext{
${ }^{1}$ This is achieved by sequentially angling the blocks away from the origin of the sPHENIX coordinate system.
} 

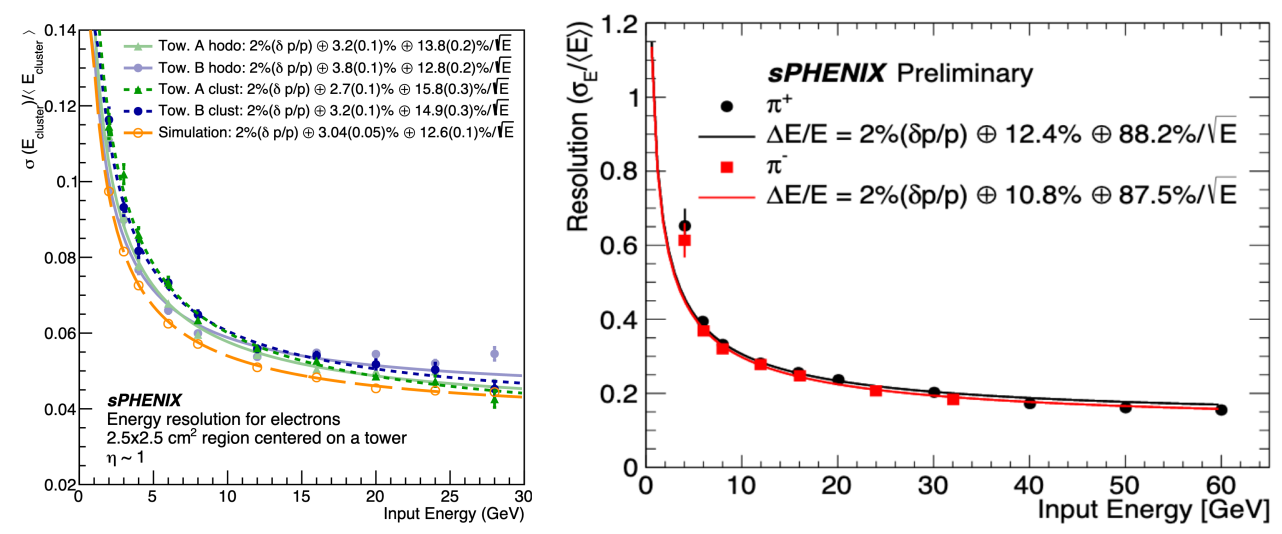

Figure 5: Measured energy resolution of the calorimeter prototypes as a function of electron input energy. Left - EMCal [7], right - HCal.

doubles as the magnetic flux return and as a support structure for the solenoid and internal detector components.

\section{Physics Goals}

The main physics goal of sPHENIX is to measure the properties of charm and beauty hadrons, including quarkonia, both through heavy flavour and jet measurements. The masses of both the $c$ and $b$-quarks are greater than $\Lambda_{Q C D}$ and $T_{Q G P}$, the former allows for the use of perturbative QCD to model the initial production while the latter excludes the thermal production of these quarks and hence the number of $c$ - and $b$-quarks produced is fixed from the initial collisions.

The STAR collaboration published the first observation of direct flow in $D^{0}-\bar{D}^{0}$ events, however the separation of the $D^{0}$ from its charge-conjugate was unable to show a significant difference between the flavours due to the initial magnetic field [8]. sPHENIX simulations indicate that any difference should be possible to measure in the $K^{-} \pi^{+}$final state, however care must be given to discern the Cabibbo-favoured $K^{-} \pi^{+}$final state from the doubly-Cabibbo suppressed and mixed $K^{+} \pi^{-}$which, if improperly accounted for, will dilute the overall effect [9]. Further, comparisons of $R_{A A}$ predictions for prompt and non-prompt $D^{0}$ 's should have comparable and experimentaldominated uncertainties respectively. sPHENIX predictions for both these measurements can be seen in Fig. 6.

Jet production at RHIC can also be used to probe QGP, with the smaller $\sqrt{s_{N N}}$ compared to that of the LHC allowing for more di-jet pairs to be found in the low $\eta$ region. sPHENIX will perform $c$ - and $b$-jet separation by exploiting the different secondary vertex masses (as the $b$-quark is significantly more massive than the $c$-quark) and use displaced vertices to tag the heavy flavour jets.

\section{Conclusion}

The sPHENIX experiment at RHIC will act both in complement to the physics program of the LHC and as a probe of regions that are inaccessible by other accelerators. It has a comprehensive 

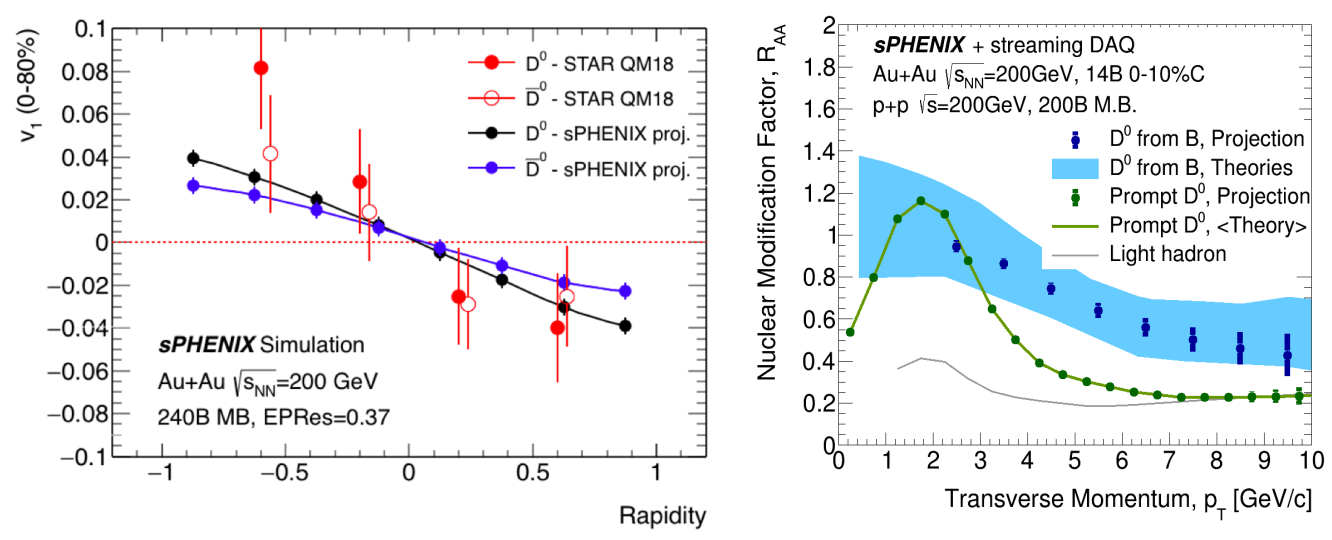

Figure 6: Left - Comparison of directed flow measurements of $D^{0}$ and $\bar{D}^{0}$ by STAR (in red) and sPHENIX simulations (in blue and black). Right - $R_{A A}$ predictions for $D^{0}$ from prompt (in green) and non-prompt (in blue) production mechanisms.

physics program involving charm hadrons while also expanding RHIC program to precision probes of beauty hadrons in a heavy ion environment, including the first separation of $\Upsilon(\mathrm{nS})$ at RHIC. The construction of the detector is progressing rapidly with commissioning occurring in 2021 and 2022 with the first data taking period scheduled for 2023.

\section{References}

[1] PHENIX Collaboration, An Upgrade Proposal from the PHENIX Collaboration, arXiv:1501.06197.

[2] H. Hahn et al., The RHIC design overview, NIM A 499 (2003) 245-263.

[3] BaBar Collaboration, BaBar Technical Design Report, Chapter 9 - Magnet Coil and Flux Return UCRL-11.L126261.

[4] ALICE Collaboration, ALPIDE, the Monolithic Active Pixel Sensor for the ALICE ITS upgrade, NIM A 824 (2016) 434-438.

[5] H. Klest, Overview and design of the sPHENIX TPC, J. Phys.: Conf. Ser.1498012025.

[6] J. Adolfsson et al., SAMPA Chip: the New 32 Channels ASIC for the ALICE TPC and MCH Upgrades, JINST 12 C04008.

[7] C.A. Aidala et al., Design and Beam Test Results for the 2D Projective sPHENIX Electromagnetic Calorimeter Prototype, arXiv:2003.13685.

[8] STAR Collaboration, First Observation of the Directed Flow of $D^{0}$ and $\bar{D}^{0}$ in Au+Au Collisions at $\sqrt{s_{N N}}=200 \mathrm{GeV}$, PRL 123, 162301 .

[9] FOCUS Collaboration, Measurement of the doubly Cabibbo suppressed decay $D^{0} \rightarrow K^{+} \pi^{-}$ and a search for charm mixing, Phys. Lett. B 618 (2005) 23-33. 